\title{
Points, lines and diamonds: a two-sorted modal logic for projective planes
}

\author{
Yde Venema*
}

\begin{abstract}
We introduce a modal language for talking about projective planes. This language is two-sorted, containing formulas to be evaluated at points and at lines, respectively. The language has two diamonds whose intended accessibility relations are the two directions of the incidence relation between points and lines.

We provide a sound and complete axiomatization for the formulas that are valid in the class of projective planes. We also show that it is decidable whether a given formula is satisfiable in a projective plane, and we characterize the computational complexity of this satisfaction problem.
\end{abstract}

${ }^{*}$ Institute of Logic, Language and Information, University of Amsterdam, Plantage Muidergracht 24, 1018 TV Amsterdam, The Netherlands. yde@wins. uva.nl The research of the author has been made possible by a fellowship of the Royal Netherlands Academy of Arts and Sciences 


\section{Introduction}

Compared to temporal logics, modal logics of space have received very little attention. I can see two reasons for this. First, temporal logic has its roots in the semantics of natural language; here, the notion of tense naturally leads to an extension of classical logics with temporal modal operators. In most familiar languages spatial concepts seem to play a less pervasive role, notwithstanding the many expressions that could be interpreted as spatial modalities. Also, the development of temporal logic has been boosted by concerns from computer science, in the context of program specification and verification. Here temporal properties are of greater interest than spatial ones.

A second reason is perhaps that it is more evident which ontologies to employ when formalizing the notion of time; apart from some notable exceptions, the standard temporal structure consists of a set of time points together with some kind of ordering of these points. When devising a formal model of space we seem to be faced with a far greater choice. Even if we decide to restrict ourselves to points as spatial objects, there are a great number of interesting relations to consider, such as nearness, collinearity, betweenness or equidistance. But also, the restriction to points as the sole entities of the mathematical model is more debatable than in the temporal case. For, space is inhabited by various kinds of things, such as lines, spheres, planes, poyhedra, etc.

Nevertheless, in recent years the interest seems to be growing in the use of modal logics for qualitative reasoning about spatial relations between objects. This increased interest stems from intended applications in the areas of Knowledge Representation and the theory of Geographical Information Systems. It is not our intention here to survey the variety of these recently developed modal logics of space - the reader is referred to LEMON [4] or LEMON \& PRATT [5]. In any case, it is clear that as yet, no consensus has been reached as to what the modal logic of space would be.

The aim of this paper is to take a class of very simple structures, a very simple modal language to talk about it, and to give a detailed account of the arising modal logic. In this way we hope to arrive at one sort of basic spatial logic, in the same way that S5 and $\mathrm{K}$ are basic modal logics, or $\mathrm{K}_{t}$ a basic temporal one.

Now projective planes are probably the simplest spatial structures around (in our account we have based ourselves on the treatment in HEYTing [3] but the facts on projective geometries used here can be found in any textbook on geometry). There seem to be two kinds of approaches to projective planes: they are either formalized as what we call collinearity frames, that is, structures consisting of points related by a ternary collinearity relation satisfying certain properties; or as two-sorted structures consisting of points and lines related by a binary incidence relation.

We take the second approach here since it seems to be the simplest. Also, since more complex spatial ontologies may be inhabited by various creatures, it seems quite natural for spatial logicians to get acquainted with many-sorted modal logics. In fact, we want to make a case for sorted modal logics as description languages for spatial structures. In the next section, we define a projective plane to be a two-sorted structure $\mathcal{F}=(P, L, I)$ with $P$ and $L$ being two disjoint sets, of points and lines, respectively; and $I \subseteq P \times L$ an incidence relation satisfying some simple conditions. It naturally follows that our corresponding modal 
language $M L G_{2}$ is two-sorted as well: we will distinguish point formulas and line formulas. The language then will have two diamonds with respectively the incidence relation and its converse as accessibility relation. These diamonds thus turn respectively line formulas into point formulas, and vice versa. Apart from some obvious adaptations, this sortedness can be incorporated in the general framework of modal logic quite smoothly.

The main results of the paper are the following. Theorem 4.2 is a completeness result: in Definition 4.1 we provide a strongly sound and complete axiom system for the two-sorted modal logic of projective planes. Theorem 5.1 states that it is decidable whether a given $M L G_{2}$-formula is satisfiable in a projective plane. Finally, Theorem 6.1 is a complexity result; we prove that the satisfaction problem for the class of projective planes is NEXPTIMEcomplete.

There are a few papers in the literature that are closely related to this approach. I got interested in the subject through a paper BALBIANI ET ALII [2], which also takes two-sorted geometrical structures as a basis. The main difference is that Balbiani cum suis wish to stay within the framework of one-sorted modal logic. Their approach is to turn two-sorted structures into multi-dimensional one-sorted structures; the 'possible worlds' in their modal structures are so-called 'tips', that is, pairs consisting of a point and a line. The price they have to pay for this is a relatively involved axiomatization and an (as yet) open decidability problem. A modal logic of one-sorted projective geometries (of arbitrary but fixed finite dimension) is developed in STEBLETsova [8]. Here the earlier mentioned collinearity frames are the basic ontologies. Stebletsova's prime aim is to find axiomatizations for classes of socalled Lyndon algebras; these are relation algebras arising as the complex algebras of structures induced by the collinearity frames. Third, when preparing the final version of this manuscript, I learned that independently, $\mathrm{Ph}$. Balbiani has obtained some of the results reported on in this paper. The reader may find his account in BALBIANI [1].

Finally, there are some obvious directions for further research that we hope to report on in the future. For instance, in this paper we only consider the binary relation of incidence, but it would be interesting to consider modal logics of richer geometrical structures; immediate candidates are the relations of parallelism, orthogonality and betweenness. Another road would lead to a study of the modal logic of projective geometries of higher dimensions; here we might stick to a two-sorted modal logic of points and lines, but in a fixed finite dimension, say $n$, it seems more natural to consider $n$-sorted modal logics.

Overview In the next section we formally introduce the syntax and semantics of our modal language $M L G_{2}$. Section 3 is about quasi-planes; these are two-sorted structures that look like projective planes but are a bit easier to work with; they play an important technical role in this paper. In section 4 we define a Hilbert style axiom system AXP, and we state and prove this system to be strongly sound and complete with respect to the class PP of projective planes. The short section after that concerns the decidability of $M L G_{2}$ with respect to the class PP. Section 6 is about complexity issues: we show that the problem whether an $M L G_{2^{-}}$ formula is satisfiable in a projective plane, is NEXPTIME-complete. Finally, in the last section we sketch some directions for further research. 
Acknowledgements I am indebted to V. Stebletsova for discussions on the modal logic of geometries (and for the $\mathrm{AT}_{\mathrm{E}} \mathrm{X}$-code producing the picture of Pappus' Theorem), and to M. Marx for help in proving the complexity result.

\section{The modal logic of projective planes}

In this section we introduce projective planes and our two-sorted modal logic $M L G_{2}$ for talking about them. Let us first see what kind of structures projective planes should be.

Definition 2.1 A two-sorted frame is a two-sorted structure $\mathcal{F}=(P, L, I)$ such that $P \cap$ $L=\varnothing$ and $I \subseteq P \times L$. Elements of $P$ and $L$ are called points and lines respectively; $I$ is called the incidence relation.

As variables ranging over points we will use $s, t, u, \ldots$; for lines we use $k, l, m, \ldots$, while $x, y, z$ will range over both kinds of objects. If the relation $I$ holds for a point $s$ and a line $k$ (notation: $s I k$ ), we will say that $s$ and $k$ are incident, but we will also use geometrically inspired terminology such as ' $s$ lies on $k$ ' or ' $k$ goes through $s$ '. Such terminology will also be employed when referring to more complex constellations; for instance ' $k$ connects $s$ and $t$ ' means that $k$ is incident with both $s$ and $t$, etc.

We will usually drop the adjective 'two-sorted' when referring to two-sorted frames. In general, we will often be rather implicit concerning sortedness when giving definitions; when employing the key word 'well-sorted' we trust the reader will be able to supply the necessary details. For instance, we will call a map $f: P \cup L \rightarrow P^{\prime} \cup L^{\prime}$ well-sorted if $f$ maps points to points and lines to lines.

Definition 2.2 A two-sorted frame $\mathcal{F}=(P, L, I)$ is called a projective plane if it satisfies

(P1) each pair of distinct points is connected by exactly one line,

(P2) each pair of distinct lines intersects in exactly one point,

(P3) there are at least four points such that no three of them are incident with one and the same line.

We let PP denote the class of projective planes.

We obtain an equivalent definition if we replace P3 with

$(P 3)^{d}$ there are at least four lines such that no three of them are incident with one and the same point.

As a consequence, we may replace the words 'point' and 'line' in any theorem concerning projective planes, and obtain another theorem. This well-known duality principle will be used frequently in this paper in order to shorten proofs.

We will now introduce the sorted modal language for frames. 
Definition 2.3 The alphabet of $M L G_{2}$ consists of the connectives $\neg,\langle 01\rangle,\langle 10\rangle$ (unary) and $\wedge$ (binary) and brackets; there are also two disjoint, countably infinite sets $V A R_{p}$ of point variables: $p, q, r, \ldots$, and $V A R_{l}$ of line variables: $a, b, c, \ldots$

The sets $\mathrm{FOR}_{p}$ and $\mathrm{FOR}_{l}$ of point- and line formulas of this language are defined by the following schema:

$$
\begin{array}{ll|l|l|l}
\sigma & :=x & \neg \sigma & \sigma \wedge \sigma & \langle 01\rangle \alpha \\
\alpha & :=a & \neg \alpha & \alpha \wedge \alpha & \langle 10\rangle \sigma
\end{array}
$$

As (meta-)variables ranging over point formulas we use $\sigma, \tau, \ldots$; for line formulas we use $\alpha, \beta, \ldots$, while for arbitrary formulas we use $\varphi, \psi, \ldots$ For sets of formulas we will use the corresponding capital Greek letters; unless explicitly stated, we always assume that sets of formulas contain formulas of one sort only. We could and perhaps should have introduced boolean connectives in sorts as well; it seems however that no confusion is likely to arise with respect to the sort of a given formula. The notation for the diamonds can be easily memorized as follows: $\langle i j\rangle \varphi$ holds at an object of dimension $i$ if it is incident with an object of dimension $j$ where $\varphi$ holds.

We can now define the semantics of our two-sorted modal logic.

Definition 2.4 Let $\mathcal{F}=(P, L, I)$ be some frame. A valuation on $\mathcal{F}$ is a map assigning subsets of $P$ to point variables and subsets of $L$ to line variables. A (two-sorted) model is a pair $\mathcal{M}=(\mathcal{F}, V)$ such that $\mathcal{F}$ is a frame and $V$ is a valuation. Given a model $\mathcal{M}=(\mathcal{F}, V)$, we define the notion of truth as follows:

$$
\begin{aligned}
& \mathcal{M}, s \Vdash p \quad \text { if } \quad s \in V(p) \text {, } \\
& \mathcal{M}, k \Vdash a \quad \text { if } \quad k \in V(a) \text {, } \\
& \mathcal{M}, x \Vdash \neg \varphi \quad \text { if } \quad \operatorname{not} \mathcal{M}, x \Vdash \varphi \text {, } \\
& \mathcal{M}, x \Vdash \varphi \wedge \psi \quad \text { if } \quad \mathcal{M}, x \Vdash \varphi \text { and } \mathcal{M}, x \Vdash \psi \text {, } \\
& \mathcal{M}, s \Vdash\langle 01\rangle \alpha \quad \text { if } \quad \text { there is some } k \text { with sIk and } \mathcal{M}, k \Vdash \alpha \text {, } \\
& \mathcal{M}, k \Vdash\langle 10\rangle \sigma \quad \text { if } \quad \text { there is some } s \text { with sIk and } \mathcal{M}, s \Vdash \sigma \text {. }
\end{aligned}
$$

The notions of satisfiability, validity and consequence are defined and denoted as usual. For instance, let $\mathrm{K}$ be some class of frames, $\Phi$ a set of formulas, and $\varphi$ a formula. Then we say that $\varphi$ is a $\mathrm{K}$-consequence of $\Phi$, notation: $\Phi={ }_{\mathrm{K}} \varphi$, if for every model $\mathcal{M}$ based on a frame in $\mathrm{K}$ and every object $x$ in $\mathcal{M}, \mathcal{M}, x \Vdash \Phi$ only if $\mathcal{M}, x \Vdash \varphi$.

In our definition of the consequence relation we require $\Phi$ and $\varphi$ to be of the same sort. This is because we are working in the local paradigm: suppose that we investigate whether $\Phi \models_{\mathrm{K}} \varphi$. We then have to check, for each $\mathrm{K}$-based model $\mathcal{M}$ and object $x$ in $\mathcal{M}$ where $\Phi$ holds, whether $\varphi$ holds at this particular object $x$. This would not make sense if $\varphi$ were of a different sort than $\Phi$. Using the global paradigm however, we would define $\Phi \models_{\mathrm{K}}^{g} \varphi$ iff for every K-based model $\mathcal{M}, \mathcal{M}=\Phi$ only if $\mathcal{M}=\varphi$; obviously, here one could lift the restriction that $\Phi$ and $\varphi$ be of the same sort. 
As abbreviations we use the operators $\langle\cdot\rangle$ and $\langle-\rangle$, with the following definitions:

$$
\begin{aligned}
\langle\cdot\rangle \sigma & \equiv\langle 01\rangle\langle 10\rangle \sigma \\
\langle-\rangle \alpha & \equiv\langle 10\rangle\langle 01\rangle \alpha .
\end{aligned}
$$

It is easy to see that in a projective plane, these operators behave like universal diamonds for points and lines, respectively.

$$
\begin{array}{cc}
\mathcal{M}, s \Vdash\langle\cdot\rangle \sigma & \text { if } \quad \text { there is some point } t \text { with } \mathcal{M}, t \Vdash \sigma, \\
\mathcal{M}, k \Vdash\langle-\rangle \alpha & \text { if } \quad \text { there is some line } l \text { with } \mathcal{M}, l \Vdash \alpha .
\end{array}
$$

It is rather straightforward to develop the model theory of sorted modal logic: notions like bisimulations or disjoint unions can be easily generalized to the two-sorted case. As an example, we provide the notion of a bounded morphism.

Definition 2.5 Let $\mathcal{F}=(P, L, I)$ and $\mathcal{F}^{\prime}=\left(P^{\prime}, L^{\prime}, I^{\prime}\right)$ be two frames, and let $f$ be a wellsorted map between their universes. This map is called a bounded morphism if it satisfies the following conditions:

(forth) $f$ is a homomorphism, that is: sIk only if $f(s) I^{\prime} f(k)$,

(back) 1. If $f(s) I^{\prime} k^{\prime}$ then there is some $k$ with sIk and $f(k)=k^{\prime}$,

2. If $s^{\prime} I^{\prime} f(k)$ then there is some $s$ with sIk and $f(s)=s^{\prime}$.

If $f$ is a surjective bounded morphism from $\mathcal{F}$ to $\mathcal{F}^{\prime}$, we say that $\mathcal{F}^{\prime}$ is a bounded morphic image of $\mathcal{F}$, notation $f: \mathcal{F} \rightarrow \mathcal{F}^{\prime}$, or $\mathcal{F} \rightarrow \mathcal{F}^{\prime}$ if we do not want to mention the map explicitly.

It is straightforward to prove the following proposition.

Proposition 2.6 Let $\mathrm{K}$ be a class of frames and $\mathbb{H}_{f} \mathrm{~K}$ the class of bounded morphic images of frames in $\mathrm{K}$. Then for any set $\Phi$ of formulas and any formula $\varphi$ we have

$$
\Phi \models_{\mathrm{K}} \varphi \text { iff } \Phi \models_{\mathbb{H}_{f} \mathrm{~K}} \varphi \text {. }
$$

Finally, simple as the language $M L G_{2}$ may seem, there are already some interesting geometrical properties that can be expressed in it. As an example we mention Pappus' theorem, a well-known concept in projective geometry.

In the formulation of Pappus' theorem below, we let $($ for $s \neq t), \overline{s t}$ denote the unique line connecting $s$ and $t$. Consider also the picture below.

Pappus Let $k$ and $l$ be distinct lines; let $t_{1}, t_{2}, t_{3}$ and $u_{1}, u_{2}, u_{3}$ be distinct points such that each $t_{i}$ and no $u_{j}$ is incident with $k$, and each $u_{i}$ and no $t_{j}$ is incident with $l$. Let (for each $i=1,2,3) v_{i}$ be the point incident with the lines $\overline{s_{j} t_{k}}$ and $\overline{s_{k} t_{j}}$ (here $j$ and $k$ are such that $\{i, j, k\}=\{1,2,3\})$. Then there is a line $m$ incident with $v_{1}, v_{2}$ and $v_{3}$. 


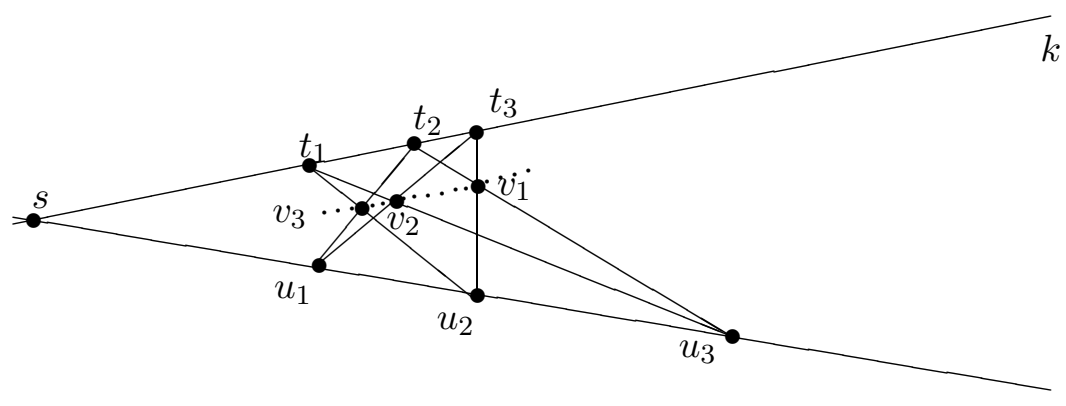

While Pappus' Theorem is a classic theorem of standard Euclidean geømetry, there are projective geometries for which it does not hold. Therefore, in the sequel we will no longer refer to Pappus' theorem but rather call a projective plane Pappian if it satisfies the property described above.

We will now define a modal formula pappus that characterizes the Pappian planes (within the class of projective planes). First consider the following abbreviations.

$$
\begin{aligned}
\operatorname{distinct}\left(\sigma_{1} \ldots \sigma_{n}\right) & :=[\cdot] \bigwedge_{i \neq j}\left(\sigma_{i} \rightarrow \neg \sigma_{j}\right), \\
\operatorname{on}\left(\alpha_{1} \ldots \alpha_{n}\right) & :=\bigwedge_{i}\langle 01\rangle \alpha_{i}, \\
\operatorname{thru}\left(\sigma_{1} \ldots \sigma_{n}\right) & :=\bigwedge_{i}\langle 10\rangle \sigma_{i}, \\
\operatorname{nthru}\left(\sigma_{1} \ldots \sigma_{n}\right) & :=\bigwedge_{i} \neg\langle 10\rangle \sigma_{i}, \\
\operatorname{meet}\left(\sigma_{1} \sigma_{2}\right)\left(\tau_{1} \tau_{2}\right) & :=\operatorname{on}\left(\operatorname{thru}\left(\sigma_{1} \sigma_{2}\right), \operatorname{thru}\left(\tau_{1} \tau_{2}\right)\right),
\end{aligned}
$$

The formulas distinct $\left(\sigma_{1} \ldots \sigma_{n}\right)$, on $\left(\alpha_{1} \ldots \alpha_{n}\right)$ and $\operatorname{meet}\left(\sigma_{1} \sigma_{2}\right)\left(\tau_{1} \tau_{2}\right)$ are point formulas; $\operatorname{thru}\left(\sigma_{1} \ldots \sigma_{n}\right)$ and $\operatorname{nthru}\left(\sigma_{1} \ldots \sigma_{n}\right)$ are line formulas. The meaning of the formula distinct $\left(\sigma_{1} \ldots \sigma_{n}\right)$ is that no $\sigma_{i}$ and $\sigma_{j}$ can be true at the same point; on $\left(\alpha_{1} \ldots \alpha_{n}\right)$ holds at those points that are incident with lines where $\alpha_{1}, \ldots, \alpha_{n}$ are true, respectively. Likewise, $\operatorname{thru}\left(\sigma_{1} \ldots \sigma_{n}\right)$ holds at those lines that go through a $\sigma_{i}$ point for each $i$; $\operatorname{nthru}\left(\sigma_{1} \ldots \sigma_{n}\right)$ holds at a line if it does not go through a $\sigma_{i}$ point for any $i$; note that $\operatorname{nthru}\left(\sigma_{1} \ldots \sigma_{n}\right)$ is not the negation of $\operatorname{thru}\left(\sigma_{1} \ldots \sigma_{n}\right)$. The formula meet $\left(\sigma_{1} \sigma_{2}\right)\left(\tau_{1} \tau_{2}\right)$ will be true for a point if it is incident with two lines that go through a $\sigma_{1}$ and a $\sigma_{2}$ point, and through a $\tau_{1}$ and a $\tau_{2}$ point, respectively.

Finally, let pappus be the formula

$$
\begin{aligned}
\text { pappus }:= & \left(\text { on }\left(\operatorname{thru}\left(q_{1} q_{2} q_{3}\right) \wedge \operatorname{nthru}\left(r_{1} r_{2} r_{3}\right), \operatorname{thru}\left(r_{1} r_{2} r_{3}\right) \wedge \operatorname{nthru}\left(q_{1} q_{2} q_{3}\right)\right)\right. \\
& \left.\wedge \operatorname{distinct}\left(q_{1} q_{2} q_{3} r_{1} r_{2} r_{3}\right)\right) \\
& \rightarrow\langle\cdot\rangle\langle 01\rangle \operatorname{thru}\left(\operatorname{meet}\left(q_{1} r_{2}\right)\left(q_{2} r_{1}\right), \operatorname{meet}\left(q_{1} r_{3}\right)\left(q_{3} r_{1}\right), \operatorname{meet}\left(q_{2} r_{3}\right)\left(q_{3} r_{2}\right)\right) .
\end{aligned}
$$

Proposition 2.7 Let $\mathcal{F}$ be a projective plane. Then $\mathcal{F}$ is Pappian iff $\mathcal{F} \models$ pappus.

Proof. Let $\mathcal{F}=(P, L, I)$ be a projective plane. Assuming that the basic idea behind the proof will be clear from the suggestive notation that we just introduced, we will only supply 
the proof for the direction from left to right, confining ourselves to a proof sketch for the other direction.

Assume that $\mathcal{F}$ is Pappian. Let $V$ be a valuation and $s$ a point such that

$$
\begin{aligned}
(\mathcal{F}, V), s \Vdash & \text { on }\left(\operatorname{thru}\left(q_{1} q_{2} q_{3}\right) \wedge \operatorname{nthru}\left(r_{1} r_{2} r_{3}\right), \operatorname{thru}\left(r_{1} r_{2} r_{3}\right) \wedge \operatorname{nthru}\left(q_{1} q_{2} q_{3}\right)\right) \\
& \wedge \operatorname{distinct}\left(q_{1} q_{2} q_{3} r_{1} r_{2} r_{3}\right) .
\end{aligned}
$$

It is straightforward to verify that this means the existence of two lines $k$ and $l$ through $s$ and six distinct points $t_{1}, t_{2}, t_{3}, u_{1}, u_{2}$ and $u_{3}$ such that $t_{i} \Vdash q_{i}$ for each $i, u_{j} \Vdash r_{j}$ for each $j$, each $t_{i}$ is incident with $k$, no $u_{i}$ is incident with $k$, each $u_{i}$ is incident with $l$ and no $t_{i}$ is incident with $l$. From this it follows that $k$ and $l$ must be distinct.

If we define $v_{1}, v_{2}$ and $v_{3}$ as in the formulation of Pappus' property, we see immediately that

$$
(\mathcal{F}, V), v_{i} \Vdash \operatorname{meet}\left(p_{j} q_{k}\right)\left(p_{k} q_{j}\right)
$$

for each $i, j$ and $k$ with $\{i, j, k\}=\{1,2,3\}$. Since $\mathcal{F}$ is Pappian, there is a line $m$ through $v_{1}$, $v_{2}$ and $v_{3}$; for this $m$ we have

$$
(\mathcal{F}, V), m \Vdash \operatorname{thru}\left(\operatorname{meet}\left(q_{1} r_{2}\right)\left(q_{2} r_{1}\right), \operatorname{meet}\left(q_{1} r_{3}\right)\left(q_{3} r_{1}\right), \operatorname{meet}\left(q_{2} r_{3}\right)\left(q_{3} r_{2}\right)\right)
$$

This immediately gives

$$
(\mathcal{F}, V), s \Vdash\langle\cdot\rangle\langle 01\rangle \operatorname{thru}\left(\operatorname{meet}\left(q_{1} r_{2}\right)\left(q_{2} r_{1}\right), \operatorname{meet}\left(q_{1} r_{3}\right)\left(q_{3} r_{1}\right), \operatorname{meet}\left(q_{2} r_{3}\right)\left(q_{3} r_{2}\right)\right)
$$

For the other direction, suppose that $\mathcal{F}$ is not Pappian. In other words, there must be $k$, $l, t_{1}, t_{2}, t_{3}, u_{1}, u_{2}, u_{3}, v_{1}, v_{2}$ and $v_{3}$ as in the formulation of Pappus' property, while there is no line through $v_{1}, v_{2}$ and $v_{3}$. Define the valutation $V$ as follows:

$$
\begin{aligned}
V\left(q_{i}\right) & :=\left\{t_{i}\right\} \\
V\left(r_{i}\right) & :=\left\{u_{i}\right\}
\end{aligned}
$$

We leave it to the reader to verify that

$$
(\mathcal{F}, V), s \Downarrow \text { pappus, }
$$

$s$ being the point where $k$ and $l$ intersect.

QED

\section{Quasi-planes}

In this paper an important role is played by a class of frames that look rather like projective planes but are a bit easier to work with.

Definition 3.1 A two-sorted frame $\mathcal{F}=(P, L, I)$ is called a quasi-plane if it satisfies

(Q1) any two given points are incident with at least one line,

(Q2) any two given lines are incident with at least one point. 
We let QP denote the class of quasi-planes.

It is obvious that every projective plane is a quasi-plane; conversely, the importance of quasi-planes lies in the fact that every quasi-plane is the bounded morphic image of some projective plane.

Theorem 3.2 Every quasi-plane is the bounded morphic image of some projective plane. As a consequence, we have that

$$
\mathrm{QP}=\mathbb{H}_{f} \mathrm{PP}
$$

By Proposition 2.6 this means that the modal logic of the classes PP and QP is exactly the same. We will make good use of this fact later on. The remainder of this section is devoted to the proof of Theorem 3.2.

Proof. The crucial idea behind the proof of Theorem 3.2 is as follows. Given a quasi-plane $\mathcal{F}^{\prime}$, we will construct its bounded pre-image and the bounded morphism in a step-by-step construction. In each step of the construction we are dealing with an approximation of these in the form of a so-called coherent network. A network consists of a two-sorted frame $\mathcal{F}$ and a well-sorted map $f$ to the universes of $\mathcal{F}^{\prime}$. Roughly speaking, such a network will be called coherent if $\mathcal{F}$ and $f$ satisfy the universal conditions of the definition of a projective plane and a bounded morphism to $\mathcal{F}^{\prime}$. It need not satisfy all the existential conditions, but our construction will be such that each of its shortcomings in this respect will eventually be taken away.

We need some terminology. Let $\mathcal{F}^{\prime}=\left(P^{\prime}, L^{\prime}, I^{\prime}\right)$ be some quasi-plane. An $\mathcal{F}^{\prime}$-network is a quadruple $\mathcal{N}=(P, L, I, f)$ such that $(P, L, I)$ is a frame, the underlying frame of $\mathcal{N}$, and $f$, the map of $\mathcal{N}$, is a well-sorted function mapping $P$ to $P^{\prime}$ and $L$ to $L^{\prime}$. The underlying frame of a network $\mathcal{N}$ is denoted by $\mathcal{F}_{\mathcal{N}}$, its map by $f_{\mathcal{N}}$.

A network $\mathcal{N}=(P, L, I, f)$ is called coherent if it satisfies

$(\mathrm{C} 0)(P \cup L, I)$ is connected as a graph,

(C1) $f$ is a homomorphism,

(C2) two distinct lines cannot both go through two distinct points.

It is called saturated if it satisfies

(S1) $f$ satisfies the back conditions of Definition 2.5,

(S2) any two given points are incident with at least one line,

(S3) any two given lines are incident with at least one point.

A network is called perfect if it is both coherent and saturated.

The following Lemma states that perfect networks extending a certain subnetwork satisfying the third condition on projective planes, contain a projective plane and a surjective bounded morphism from this plane to the target quasi-plane of the network. 
Lemma 3.3 Let $\mathcal{N}$ be a perfect $\mathcal{F}^{\prime}$-network. Assume that $\mathcal{F}_{\mathcal{N}}$ also satisfies P3. Then $\mathcal{F}_{\mathcal{N}}$ is a projective plane and $f_{\mathcal{N}}: \mathcal{F}_{\mathcal{N}} \rightarrow \mathcal{F}^{\prime}$.

Proof. It is easy to see that $\mathcal{F}_{\mathcal{N}}$ must be a projective plane: it satisfies $\mathrm{P} 1$ by $\mathrm{S} 2$ and $\mathrm{C} 2$, $\mathrm{P} 2$ by $\mathrm{S} 3$ and $\mathrm{C} 2$, and $\mathrm{P} 3$ by assumption. It is likewise easy to prove that $f_{\mathcal{N}}$ is a bounded morphism: $\mathrm{S} 1$ and $\mathrm{C} 1$ are the back- and forth conditions, respectively.

The only potential difficulty is to show that $f_{\mathcal{N}}$ is surjective. Let $s^{\prime}$ be an arbitrary point in $\mathcal{F}^{\prime}$. Consider some point $t$ in $P$ (the set of points of $\mathcal{N}$ ). It follows from Q1 that $f_{\mathcal{N}}(t) I^{\prime} k^{\prime}$ and $s^{\prime} I^{\prime} k^{\prime}$ for some line $k^{\prime}$ in $L^{\prime}$. But then two applications of S1 reveal the existence of a line $k$ and a point $s$ such that $t I k, f_{\mathcal{N}}(k)=k^{\prime}$ and $s I k, f_{\mathcal{N}}(s)=s^{\prime}$. In other words, we have found the required pre-image of $s^{\prime}$ under $f_{\mathcal{N}}$. The case for an arbitrary line in $\mathcal{F}^{\prime}$ is likewise. (In fact we have proved that any bounded morphism to a quasi-plane is surjective.) QED

Hence, we have reduced the task of finding a surjective bounded morphism onto $\mathcal{F}^{\prime}$ to a quest for a perfect $\mathcal{F}^{\prime}$-network. The existence of such an object is given by the following Lemma.

Lemma 3.4 For any quasi-plane there is a perfect network satisfying P3.

Proof. The key idea underlying the proof of Lemma 3.4 is that any witness to the imperfection of a coherent network can be removed. Such witnesses will be called defects and come in kinds, according to the saturation condition that is violated. For instance, an S1-defect of an $\mathcal{F}^{\prime}$-network $\mathcal{N}=(P, L, I, f)$ consists of (a) a point $s$ in $P$ and a line $k^{\prime}$ in $L^{\prime}$ such that $f(s) I^{\prime} k^{\prime}$ while there is no $k$ in $L$ such that $s I k$ and $f(k)=k^{\prime}$, or (b) a line $k$ in $L$ and a point $s^{\prime}$ in $P^{\prime}$ such that $s^{\prime} I^{\prime} f(k)$ while there is no $s$ in $P$ such that $s I k$ and $f(s)=s^{\prime}$. S2- and S3-defects have similar definitions.

Then, we have to express more precisely what it means to repair a defect. Here we need the notion of one network extending another. Let $\mathcal{N}_{0}$ and $\mathcal{N}_{1}$ be two $\mathcal{F}^{\prime}$-networks. We say that $\mathcal{N}_{1}$ extends $\mathcal{N}_{0}$, notation: $\mathcal{N}_{0} \triangleleft \mathcal{N}_{1}$, if $P_{0}$ and $L_{0}$ are subsets of $P_{1}$ and $L_{1}$, respectively; $I_{0}$ is the restriction of $I_{1}$ to $P_{0} \times L_{0}$; and $f_{0}$ is the restriction of $f_{1}$ to $P_{0} \cup L_{0}$.

The following shows that defects of coherent networks can always be repaired.

Claim 1 For any defect of a coherent $\mathcal{F}^{\prime}$-network $\mathcal{N}$ there is an $\mathcal{N}^{+} \triangleright \mathcal{N}$ lacking this defect.

Proof of Claim Let $\mathcal{N}=(P, L, I, f)$ be a coherent network and assume that $\mathcal{N}$ has some defect. We will prove Claim 1 via a case distinction to the type of this defect.

S1-defects.

Assume that $f(s) I^{\prime} k^{\prime}$ for some point $s$ in $P$ and some line $k^{\prime}$ in $L^{\prime}$ such that there is no $k$ in $L$ such that $s I k$ and $f(k)=k^{\prime}$. (Repairing the other kind (b) of S1-defect will be analogous, by the principle of duality.)

Take some new element $k$ (new meaning that $k \notin P \cup L$ ) and define $\mathcal{N}^{+}$by

$$
\begin{aligned}
P^{+} & :=P, \\
L^{+} & :=L \cup\{k\}, \\
I^{+} & :=I \cup\{(s, k)\}, \\
f^{+} & :=f \cup\left\{\left(k, k^{\prime}\right)\right\} .
\end{aligned}
$$


It is clear that $\mathcal{N}^{+}$is a network that does not have the old defect of $\mathcal{N}$, and that $\mathcal{N} \triangleleft \mathcal{N}^{+}$. It is left to show that $\mathcal{N}^{+}$is coherent.

It is immediate that $\mathcal{N}^{+}$satisfies $\mathrm{C} 0$, by the definition of $I^{+}$and the coherency of $\mathcal{N}$. For $\mathrm{C} 1$, the only new case to check is whether $f^{+}(s) I^{\prime} f^{+}(k)$, but this is so by the assumption on $s$ and $k^{\prime}$. Finally, suppose that $\mathcal{N}^{+}$violates condition $\mathrm{C} 2$; by the definition of $\mathcal{N}^{+}$and the fact that $\mathcal{N}$ satisfies $\mathrm{C} 2$, this means that the new line $k$ must be involved. But $k$ cannot cause any problem since it is incident with only one point. This shows that $\mathcal{N}^{+}$satisfies all the coherency conditions.

S2-defects.

Assume that $P$ contains two distinct points $s$ and $t$ that are not connected by a line.

By Q1 there is some line $k^{\prime}$ in $L^{\prime}$ such that $f(s) I^{\prime} k^{\prime}$ and $f(t) I^{\prime} k^{\prime}$. Take some new element $k$ and define $\mathcal{N}^{+}$by

$$
\begin{aligned}
P^{+} & :=P, \\
L^{+} & :=L \cup\{k\}, \\
I^{+} & :=I \cup\{(s, k),(t, k)\}, \\
f^{+} & :=f \cup\left\{\left(k, k^{\prime}\right)\right\} .
\end{aligned}
$$

As in the previous case, it is easy to see $\mathcal{N}^{+}$is an extension of $\mathcal{N}$ in which the defect has been repaired, and that $\mathcal{N}^{+}$satisfies $\mathrm{C} 0$ and $\mathrm{C} 1$. Now suppose that the points $u$ and $v$ and the lines $l$ and $m$ constitute a counterexample to the validity of $\mathrm{C} 2$. That is, we have $u \neq v$, $l \neq m$ while both points lie on both lines. As before, the new line $k$ must be involved, say $k=m$; but then it follows from $u I k, v I k$ and $u \neq v$ that either $u=s$ and $v=t$, or $u=t$ and $v=s$. Now we look at the other line $l$, which is distinct from $k$; hence, it must be old, that is, belong to $\mathcal{N}$; but if such an old line goes through both $s$ and $t$ then $s$ and $t$ did not constitute an S2-defect of $\mathcal{N}$ after all! The conclusion is that $\mathrm{C} 2$ holds for $\mathcal{N}^{+}$, which is thus a coherent network.

S3-defects.

Assume that $L$ contains two distinct lines $k$ and $l$ that do not intersect.

These defects are the duals of S2-defect, and thus we may refer to the principle of duality for the strategy of repairing them.

This proves Claim 1.

Let us now see why there is always a coherent network satisfying P3.

Claim 2 For any quasi-plane $\mathcal{F}^{\prime}$ there is a coherent $\mathcal{F}^{\prime}$-network satisfying P3.

Proof of Claim Let $\mathcal{F}^{\prime}=\left(P^{\prime}, L^{\prime}, I^{\prime}\right)$ be some quasi-plane; take some point $s^{\prime} \in P^{\prime}$. It follows from Q1 that there is some line $k^{\prime} \in L^{\prime}$ through $s^{\prime}$. From this, it is immediate that there is some $\mathcal{F}^{\prime}$-network, say $\mathcal{N}_{0}$, containing a point $s_{0}$ which is incident with a line $k_{0}$, and with $f_{0}\left(s_{0}\right)=s^{\prime}, f_{0}\left(k_{0}\right)=k^{\prime}$. Obviously, $\mathcal{N}_{0}$ is coherent.

But now we turn to the techniques we used before in order to repair S1-defects. Note that we do not need the existence of actual defects to perform the construction used there. It is 
easy to prove the existence of an $\mathcal{F}^{\prime}$-network $\mathcal{N}=(P, L, I, f)$ extending $\mathcal{N}_{0}$ and containing distinct points $s_{1}, s_{2}, s_{3}, s_{4}$ and distinct lines $k_{1}, k_{2}, k_{3}, k_{4}$ such that $s_{i} I k_{i}$ and $s_{i+1} I k_{i}$ (all $i$ for which it makes sense), while no further incidences hold for these points and lines. But that is precisely what was required to prove Claim 2.

Now we are prepared to finish the proof of Lemma 3.4. Let $\mathcal{F}^{\prime}=\left(P^{\prime}, L^{\prime}, I^{\prime}\right)$ be a quasiplane; take some cardinal $\kappa$ larger than the size of $\mathcal{F}^{\prime}$ (which we define as the sum of the sizes of the sets $P^{\prime}$ and $L^{\prime}$ ). Using some standard combinatorics, it is possible to build a sequence $\left(\mathcal{N}_{\alpha}\right)_{\alpha<\kappa}$ of coherent $\mathcal{F}^{\prime}$-networks satisfying

1. $\mathcal{N}_{0}$ satisfies P3,

2. $\mathcal{N}_{\beta}$ extends $\mathcal{N}_{\alpha}$ whenever $\beta>\alpha$,

3. for a limit ordinal $\lambda, \mathcal{N}_{\lambda}=\bigcup_{\alpha<\lambda} \mathcal{N}_{\alpha}$ (where unions of networks are defined in the obvious way),

4. for every $\alpha$, every defect of $\mathcal{N}_{\alpha}$ will be repaired in an $\mathcal{N}_{\beta}$ for some $\beta>\alpha$.

For the first item we use Claim 2 and for the fourth item, Claim 1. We also use the fact that coherency if preserved under taking unions and that unions of networks extend the networks occurring in the union. The 'standard combinatorics', needed to make sure that every defect of a network $\mathcal{N}_{\alpha}$ will be repaired at some later stage, is completely analogous to a proof in section 2 of Venema [9].

It is then straightforward to verify that the $\mathcal{F}^{\prime}$-network $\mathcal{N}=\bigcup_{\alpha<\kappa} \mathcal{N}_{\alpha}$ is perfect and satisfies P3.

QED

Finally, it follows immediately from the Lemmas 3.3 and 3.4 that every quasi-plane is the bounded morphic image of some projective plane. This shows that QP $\subseteq \mathbb{H}_{f} \mathrm{PP}$. Since it is straightforward to verify that the opposite inclusion holds as well, this proves Theorem 3.2. QED

\section{Completeness}

In this section we define a two-sorted axiom system AXP, which we will then show to be strongly sound and complete with respect to the logic of the projective planes.

Definition 4.1 Consider the axiom system AXP, consisting of the following axioms:

$(C T)$ all classical tautologies

$(D B) \quad[01](a \rightarrow b) \rightarrow([01] a \rightarrow[01] b)$

$[10](p \rightarrow q) \rightarrow([10] p \rightarrow[10] q)$

$(C J) \quad p \rightarrow[01]\langle 10\rangle p$

$a \rightarrow[10]\langle 01\rangle a$

$\left(\mathrm{D}_{l}\right) \quad\langle 01\rangle \top$

$\left(\mathrm{D}_{p}\right) \quad\langle 10\rangle \top$

$\left(4_{\langle\cdot\rangle}\right) \quad\langle\cdot\rangle\langle\cdot\rangle p \rightarrow\langle\cdot\rangle p$.

$(4\langle-\rangle) \quad\langle-\rangle\langle-\rangle p \rightarrow\langle-\rangle p$. 
Its derivation rules are:

MP Modus Ponens

$$
\frac{\varphi \rightarrow \psi \quad \varphi}{\psi}
$$

$\mathbf{N}$ Necessitation for [01] and [10]

$$
\frac{\alpha}{[01] \alpha} \quad \frac{\sigma}{[10] \sigma}
$$

SUB Substitution for any substitution $(\cdot)^{s}$ uniformly replacing in any formula $\varphi$ some propositional variables by some formula of the same sort.

$$
\frac{\varphi}{\varphi^{s}}
$$

In our presentation of AXP we have not been very explicit about the sortedness of its structure. For instance, we just said that 'all classical tautologies' are axioms of AXP; this means that for instance, both the point formula $p \vee \neg p$ and the line formula $a \vee \neg a$ are AXPaxioms. Also, it would be more precise to make a distinction between point theorems and line theorems; in such a presentation, the rule of necessitation turns line theorems into point theorems and vice versa, while the other rules do not change the sort of the theorem.

Let us first give a brief discussion of the axioms. To start with, it is obvious that we need all classical tautologies and the distribution axioms. CJ is the conjugacy or 'converse' axiom; it forms the modal way of stating that the accessibility relations connected to the diamonds $\langle 01\rangle$ and $\langle 10\rangle$ are each other's converse. The axiom $\mathrm{D}_{l}$ makes that each point is incident with at least one line; $4_{\langle\cdot\rangle}$ is the transitivity axiom for $\langle\cdot\rangle$; it is needed to ensure that the accessibility relation connected to $\langle\cdot\rangle$ is an equivalence relation - we will see below how the conditions of reflexivity and symmetry are implicitly taken care of. Finally, the axioms $\mathrm{D}_{p}$ and $4_{\langle-\rangle}$are the obvious duals of $\mathrm{D}_{l}$ and $4_{\langle\cdot\rangle}$.

Given the sortedness of the system AXP, the notions of derivation, theorem, consistency and the like are standard. A derivation in AXP is a finite sequence $\varphi_{1}, \ldots, \varphi_{n}$ of formulas such that each formula is either an axiom or the result of applying a rule to a subset of the formulas occurring higher in the list. A formula $\varphi$ is a theorem of AXP, denoted $\vdash_{P} \varphi$, if there is a derivation of which $\varphi$ is the last item. A formula $\varphi$ is provable in AXP from a set of formulas $\Phi$, denoted $\Phi \vdash_{P} \varphi$, if there are $\varphi_{1}, \ldots, \varphi_{n} \in \Phi$ such that $\vdash_{P}\left(\varphi_{1} \wedge \ldots \wedge \varphi_{n}\right) \rightarrow \varphi$. A set of formulas $\Phi$ is consistent in AXP if $\Phi \nvdash P P_{P} \perp$. A formula $\varphi$ is consistent if $\{\varphi\}$ is. A set of formulas $\Phi$ is a maximally consistent set (MCS) if it is consistent, and any proper superset is inconsistent; MCP and MCL denote the sets of maximal consistent sets of point and line formulas, respectively.

We can now state and prove the main result of this section.

Theorem 4.2 AXP is strongly sound and complete with respect to the class PP of projective planes. That is, for any set $\Phi$ of formulas and any formula $\varphi$ (of the same sort) we have

$$
\Phi \vdash_{P} \varphi \Longleftrightarrow \Phi \models_{\mathrm{PP}} \varphi .
$$


Proof. We leave the soundness proof to the reader. For completeness, our strategy is to establish the analogous result for the class of quasi-planes; the proof will be based on the canonical frame method. Obviously, our logic will give rise to a two-sorted canonical model; apart from that, however, all definitions and properties are standard.

The canonical frame for AXP is defined as the two-sorted structure $\mathcal{F}^{c}=\left(\mathrm{MCP}, \mathrm{MCL}, I^{c}\right)$. Here the relation $I^{c}$ is given by

$$
\Sigma I^{c} \Gamma \text { iff }\langle 01\rangle \gamma \in \Sigma \text { for all } \gamma \in \Gamma .
$$

Likewise, the relations $E_{p}^{c}$ and $E_{l}^{c}$ are given by

$$
\begin{aligned}
& \Sigma E_{p}^{c} \Pi \quad \Longleftrightarrow\langle\cdot\rangle \pi \in \Sigma \text { for all } \pi \in \Pi, \\
& \Gamma E_{l}^{c} \Delta \quad \Longleftrightarrow\langle-\rangle \delta \in \Gamma \text { for all } \delta \in \Delta .
\end{aligned}
$$

We will see that $\mathcal{F}^{c}$ is in fact the disjoint union of quasi-planes. First, we need some properties of the canonical frame.

Claim 1 The canonical frame for AXP has the following properties (the formulation of each statement is to be read universally):

C1 $\Sigma I^{c} \Gamma$ iff $\langle 10\rangle \sigma \in \Gamma$ for all $\sigma \in \Sigma$.

C2 If $\langle 01\rangle \gamma \in \Sigma$ then $\gamma \in \Gamma$ for some $\Gamma$ with $\Sigma I^{c} \Gamma$.

C3 If $\langle 10\rangle \sigma \in \Gamma$ then $\sigma \in \Sigma$ for some $\Sigma$ with $\Sigma I^{c} \Gamma$.

C4 There is a $\Gamma$ with $\Sigma I^{c} \Gamma$.

C5 There is a $\Sigma$ with $\Sigma I^{c} \Gamma$.

C6 $\Sigma E_{p}^{c} \Pi$ iff $\Sigma I^{c} \Gamma$ and $\Pi I^{c} \Gamma$ for some $\Gamma$.

C7 $E_{p}^{c}$ is an equivalence relation.

C8 $\Gamma E_{l}^{c} \Delta$ iff $\Sigma I^{c} \Gamma$ and $\Sigma I^{c} \Delta$ for some $\Sigma$.

C9 $E_{l}^{c}$ is an equivalence relation.

Proof of Claim The proofs of C1-C3 are completely analogous to the standard (one-sorted) case. $\mathrm{C} 4$ follows from $\mathrm{C} 2$ since the axiom $\mathrm{D}_{l}$ belongs to every MCP; likewise, C5 follows from $\mathrm{C} 3$ and axiom $\mathrm{D}_{p}$. C6 is again standard modal logic. In order to prove $\mathrm{C} 7$, first note that $E_{p}^{c}$ is reflexive and symmetric by $\mathrm{C} 4$ and $\mathrm{C} 6$; we can easily prove transitivity using axiom $4_{\langle\cdot\rangle}$. Finally, $\mathrm{C} 8$ and $\mathrm{C} 9$ are the duals of $\mathrm{C} 6$ and $\mathrm{C} 7$, respectively.

We now concentrate on subframes of $\mathcal{F}^{c}$. Let $\Phi$ be some MCS. The canonical subframe of $\Phi$ is defined as the structure $\mathcal{F}_{\Phi}^{c}=\left(P_{\Phi}^{c}, L_{\Phi}^{c}, I_{\Phi}^{c}\right)$. Here $P_{\Phi}^{c}$ and $L_{\Phi}^{c}$ are the sets of MCPs and MCLs respectively that can be reached from $\Phi$ by a finite number of $I^{c}$-steps (moving from points to lines and back again). $I_{\Phi}^{c}$ is the relation $I^{c}$ restricted to $P_{\Phi}^{c} \cup L_{\Phi}^{c}$. 
Claim $2 \mathcal{F}^{c}$ is a disjoint union of frames $\mathcal{F}_{\Phi}^{c}$, each of which is a quasi-plane.

Proof of Claim It is obvious by the definitions that each frame $\mathcal{F}_{\Phi}^{c}$ is a generated subframe of $\mathcal{F}^{c}$; from this observation, the first part of the claim is immediate. Let $\Pi$ and $\Sigma$ be two maximal consistent point sets in $\mathcal{F}_{\Phi}^{c}$. It easily follows from the definition of $\mathcal{F}^{c}$ that there is a finite $I^{c}$-path from $\Pi$ to $\Sigma$ (simply reverse the path from $\Phi$ to $\Pi$ and concatenate the path from $\Phi$ to $\Sigma$ to it). Using C6 and C7, by induction on the length of this path we can prove that $\Pi E_{p}^{c} \Sigma$, so by $\mathrm{C} 6$ there is some $\Gamma$ with $\Pi I^{c} \Gamma$ and $\Sigma I^{c} \Gamma$. Clearly $\Gamma$ belongs to $L_{\Phi}^{c}$, so by definition, the relation holds in $\mathcal{F}_{\Phi}^{c}$ as well.

The proof that any two MCLs are joined by an MCP in $\mathcal{F}^{c}$ is the dual of the proof just given.

For each frame of the form $\mathcal{F}_{\Phi}^{c}$ we can prove a truth lemma:

Claim 3 For every maximal consistent set $\Phi$, all maximal consistent sets $\Psi$ in $\mathcal{F}_{\Phi}^{c}$ and all formulas $\psi$ :

$$
\mathcal{F}_{\Phi}^{c}, \Psi \Vdash \psi \text { iff } \psi \in \Psi .
$$

Proof of Claim By a standard formula induction.

We now finish the completeness proof, reasoning by contraposition. Assume that $\Sigma$ and $\tau$ are such that $\Sigma \nvdash_{P} \tau$ (the proof for line formulas is dual). By a standard Lindenbaum construction $\Sigma$ can be extended to a maximal consistent point set $\Pi$ not containing $\tau$. It follows from Claim 3 that $\mathcal{F}_{\Pi}^{c}, \Pi \Vdash \sigma$ for all $\sigma \in \Sigma \subseteq \Pi$, while $\mathcal{F}_{\Pi}^{c}, \Pi \Downarrow \tau$. $\mathcal{F}_{\Pi}^{c}$ is a quasi-plane by Claim 2, so we have proved that $\Sigma \not \nvdash_{\mathrm{QP}} \tau$. But then it follows from Theorem 3.2 and Proposition 2.6 that $\Sigma \not \models \mathrm{PP} \tau$.

QED

\section{Decidability}

The main result of this section states that PP has a decidable $M L G_{2}$-theory. We will prove this result by establishing the finite model property of $M L G_{2}$ with respect to the class of quasi-planes.

Theorem 5.1 It is decidable whether a given $M L G_{2}$-formula is satisfiable in a projective plane.

Let $|\varphi|$ denote the length of the formula $\varphi$; as before, the size of a frame $\mathcal{F}=(P, L, I)$ is defined as the sum of the cardinalities of $P$ and $L$. The following proposition states the strong finite model property for $M L G_{2}$ on the class of quasi-planes.

Proposition 5.2 Let $\xi$ be an $M L G_{2}$-formula. If $\xi$ is satisfiable in a projective plane (or, equivalently, in a quasi-plane), then it is satisfiable in a quasi-plane of size not exceeding $2^{1+|\xi|}$. 
Proof. The proof of this proposition is by a straightforward filtration argument. Assume that $\xi$ is an $M L G_{2}$-formula which is satisfiable in the model $\mathcal{M}=(P, L, I, V)$, with $(P, L, I)$ being a projective plane. Let $C l(\xi)$ be the set of subformulas of $\xi$; note that $C l(\xi)$ will generally contain both point and line formulas. By our notational convention, ' $\sigma \in C l(\xi)$ ' is short for ' $\sigma$ is a point formula in $C l(\xi)$ '.

Let $\equiv$ be the following relation on $P$, and on $L$, respectively:

$$
\begin{aligned}
& s \equiv t \quad \text { if } \quad \text { for all } \sigma \in C l(\xi): \quad(\mathcal{M}, s \Vdash \sigma \quad \Longleftrightarrow \quad \mathcal{M}, t \Vdash \sigma), \\
& k \equiv l \quad \text { if } \quad \text { for all } \alpha \in C l(\xi):(\mathcal{M}, k \Vdash \alpha \quad \Longleftrightarrow \mathcal{M}, l \Vdash \alpha) .
\end{aligned}
$$

Obviously, $\equiv$ is an equivalence relation; we denote the equivalence class of an object $x$ by $[x]$. Let $\bar{P}$, and $\bar{L}$ denote the set of equivalence classes of $P$ and $L$, respectively, and define $\bar{I} \subseteq \bar{P} \times \bar{L}$ by

$$
[s] \bar{I}[k] \text { if } t I l \text { for some } t \equiv s \text { and } l \equiv k .
$$

The valuation $\bar{V}$ is defined by

$$
\bar{V}(p)=\{[s] \mid s \in V(p)\},
$$

and likewise for line variables. Of course, this is only well-defined for variables in $C l(\xi)$.

Finally, the filtrated frame $\overline{\mathcal{F}}$ and model $\overline{\mathcal{M}}$ are defined as $\overline{\mathcal{F}}=(\bar{P}, \bar{L}, \bar{I})$ and $\bar{M}=(\overline{\mathcal{F}}, \bar{V})$. By a standard proof (using formula induction) we can prove that for all objects $x$ in $\mathcal{M}$ and all formulas $\varphi \in C l(\xi)$ :

$$
\mathcal{M}, x \Vdash \varphi \text { iff } \overline{\mathcal{M}},[x] \Vdash \varphi .
$$

From this it is immediate that $\xi$ is satisfiable in $\overline{\mathcal{M}}$. Clearly, the size of both $\bar{P}$ and $\bar{L}$ is bounded by the number of subsets of $C l(\xi)$. Since $C l(\xi)$ is the set of subformulas of $\xi$, its size does not exceed the length of $\xi$. It follows that the size of $\overline{\mathcal{F}}$ is at most $2 \cdot 2^{|\xi|}=2^{1+|\xi|}$.

Finally, we have to show that $\overline{\mathcal{F}}$ is a quasi-plane. Let $[s]$ and $[t]$ be points in $\overline{\mathcal{F}}$. Since $\mathcal{F}$ is a projective plane, there is a (unique) line $k$ connecting $s$ and $t$. But then $[k]$ is a line in $\overline{\mathcal{F}}$ through $[s]$ and $[t]$. The proof that any pair of lines intersect in some point, is of course dual to this proof.

QED

Proof of Theorem 5.1. In order to decide whether $\xi$ is satisfiable in a projective plane, it suffices to make a list of all models of size at most $2^{1+|\xi|}$, and to check of each such model: first, whether it is based on a quasi-plane, and second, whether $\xi$ is satisfiable in it. Both of these criteria can be checked in time polynomial in the size of the model, so we have a terminating algorithm, which is correct by Proposition 5.2.

QED

\section{Complexity}

The aim of this section is to characterize the complexity of the satisfiability problem for the class of projective planes. More precisely, let PP-SAT be the problem whether a given $M L G_{2^{-}}$ formula $\varphi$ is satisfiable in some model based on a projective plane. We will prove PP-SAT to be NEXPTIME-complete; that is, we can show that PP-SAT itself can be solved in exponential time by a non-deterministic Turing machine, and also, that every NEXPTIME-hard problem can be effectively reduced to PP-SAT. 
Theorem 6.1 PP-SAT is NEXPTIME-complete.

Proof. Immediate by the Propositions 6.2 and 6.3 below.

QED

The following proposition indicates that NEXPTIME is an upper bound for the complexity of PP-SAT.

Proposition 6.2 PP-SAT is decidable in NEXPTIME.

Proof. Immediate by Proposition 5.2: in order to calculate whether an $M L G_{2}$-formula $\xi$ is satisfiable in a projective plane, let a Turing machine non-deterministically choose a model of size not exceeding $2^{1+|\xi|}$ and calculate (in time polynomial in the size of the model) whether its underlying frame is a quasi-plane and whether $\xi$ is satisfiable in it.

QED

The second proposition indicates that NEXPTIME is an lower bound for the complexity of PP-SAT.

\section{Proposition 6.3 PP-SAT is NEXPTIME-hard.}

Proof. The basic idea behind the proof is to reduce the satisfiability problem of a certain (single sorted) poly-modal logic to PP-SAT.

Consider the (one-sorted) modal language $L_{2}$ with two diamonds $\diamond_{0}$ and $\diamond_{1}$. A frame for this language is of the form $\mathcal{F}=\left(W, R_{0}, R_{1}\right)$ in which $R_{i}$ is the accessibility relation for $\diamond_{i}$. Let $\mathrm{K}$ be the class of all frames in which $R_{0}$ and $R_{1}$ are two commuting equivalence relations. We have a special interest in a particular frame in $\mathrm{K}$, which is defined as follows. Let $\mathbb{Q}$ be the set of rational numbers, and define the relations $\equiv_{0}$ and $\equiv_{1}$ on pairs of rational numbers as follows:

$$
\left(q_{0}, q_{1}\right) \equiv_{i}\left(r_{0}, r_{1}\right) \text { iff } q_{i}=r_{i} .
$$

The frame $\left(\mathbb{Q}^{2}, \equiv_{0}, \equiv_{1}\right)$ will be called the rational square, notation: $\mathcal{S}_{\mathbb{Q}}$. Observe that as yet none of the structure of $\mathbb{Q}$ is involved here, only its cardinality. It can be proved that an $L_{2}$-formula is satisfiable in $\mathrm{K}$ iff it is satisfiable in $\mathcal{S}_{\mathbb{Q}}$. For, since $\mathrm{K}$ is elementary, an $L_{2}$-formula is satisfiable in $\mathrm{K}$ iff it is satisfiable in a countable frame $\mathcal{F}$ in $\mathrm{K}$. It can be shown via a step-by-step construction, similar to the one employed in the proof of Theorem 3.2, that every countable frame in $\mathrm{K}$ is the bounded morphic image of $\mathcal{S}_{\mathbb{Q}}$. For more details, the reader is referred to section 2.2 of in MARX \& VEnEma [7].

Now let K-SAT be the problem whether a given $L_{2}$-formula is satisfiable in a K-frame, or equivalently, in $\mathcal{S}_{\mathbb{Q}}$. $\mathrm{K}$ is known to have a NEXPTIME-complete satisfiability problem, cf. Corollary 4.14 in MARx [6]; hence, if we can find a polynomial time reduction from K-SAT to PP-SAT, we have proved NEXPTIME to be a lower bound for the complexity of PP-SAT.

For this reduction, we will define a translation $\pi$ from $L_{2}$-formulas to point formulas in $M L G_{2}$. The variables of $L_{2}$ will be considered as point variables; we will also need two line variables $b_{0}$ and $b_{1}$; as abbreviations we use $l \mathrm{~b}_{0}:=b_{0} \wedge \neg b_{1}$ and $l \mathrm{~b}_{1}:=b_{1} \wedge \neg b_{0}$. Lines where the proposition letter $b_{i}$ holds, will be called blue $i$; a line where $1 \mathrm{~b}_{i}$ holds is light blue $e_{i}$ and a line with both shades of blue is dark blue. As a further abbreviation we will use light $:=\neg\langle 01\rangle\left(b_{0} \wedge b_{1}\right)$; note that this is a point formula, which holds for points that do not 
lie on a dark blue line. Now consider the following formulas that will form conjuncts of the translation of any $L_{2}$-formula.

$$
\begin{aligned}
\text { sky } & :=[\cdot]\left(\langle 01\rangle b_{0} \wedge\langle 01\rangle b_{1}\right), \\
\text { but } & \left.:=[\cdot]\left((\langle 01\rangle] \mathrm{b}_{0} \wedge\langle 01\rangle \mathrm{lb}_{1}\right) \rightarrow \text { light }\right) .
\end{aligned}
$$

The first of these states that any point lies on a blue $i$ line of each shade, the second means that if a point is incident with a light blue line of each shade, it cannot be incident with a dark blue line as well.

Furthermore, we use, for a point formula $\sigma$,

$$
\langle\cdot\rangle_{i} \sigma:=\langle 01\rangle\left(\mathrm{Ib}_{i} \wedge\langle 10\rangle(\text { light } \wedge \sigma)\right) .
$$

This formula will be true at a point $s$ if $s$ 'sees a light $\sigma$-point along a light blue ${ }_{i}$ line'. Now consider the formula

$$
\mathrm{bb}(\sigma):=\bigwedge_{i}\left(\left(\text { light } \wedge\langle\cdot\rangle_{i} \sigma\right) \rightarrow[01]\left(\mathrm{lb}_{i} \rightarrow\langle 10\rangle(\text { light } \wedge \sigma)\right) .\right)
$$

If this formula holds in a point $s$, it means that whenever $s$ is light and sees a light $\sigma$ point along some light blue line, it sees some light $\sigma$ point along each light blue line of the same shade.

We are now ready for the definition of the translation function. First consider the following map (.) $)^{+}$from $L_{2}$-formulas to $M L G_{2}$-formulas.

$$
\begin{aligned}
p^{+} & :=p, \\
(\neg \varphi)^{+} & :=\neg \varphi^{+}, \\
(\varphi \wedge \psi)^{+} & :=\varphi^{+} \wedge \psi^{+} . \\
\left(\diamond_{i} \varphi\right)^{+} & :=\langle\cdot\rangle_{i} \varphi^{+} .
\end{aligned}
$$

Finally, the translation function $\pi$ is defined as

$$
\pi(\xi):=\xi^{+} \wedge \text { light } \wedge \text { sky } \wedge \text { but } \wedge \bigwedge_{\varphi \in C l(\xi)}[\cdot] \mathrm{bb}\left(\varphi^{+}\right) .
$$

Here $C l(\xi)$ is the set of subformulas of $\xi$; note that we are talking $L_{2}$-formulas here. Observe that the length of $\varphi^{+}$is linear in the length of $\varphi$, and the length of $\operatorname{bb}(\sigma)$ is linear in the length of $\sigma$. Hence, since the number of formulas in $C l(\xi)$ is linear in the length of $\xi, \pi(\xi)$ can be computed in quadratic time. Hence, it follows from the next two claims that K-SAT is polynomial time reducible to PP-SAT.

Claim 1 If an $L_{2}$-formula $\xi$ is satisfiable in $\mathcal{S}_{\mathbb{Q}}$, then $\pi(\xi)$ is satisfiable in PP.

Proof of Claim Assume that $\xi$ is satisfiable in the rational square, say in the model $\mathcal{M}=\left(\mathcal{S}_{\mathbb{Q}}, V\right)$. We will transform this model into an $M L G_{2}$-model which is based on a projective plane. 
Let us first give an intuitive explanation of the proof idea. The basis of our transformation is the observation that the frame $\mathcal{S}_{\mathbb{Q}}$ itself already looks very much like a plane; the accessibility relations $\equiv_{0}$ and $\equiv_{1}$ can be seen as collections of vertical and horizontal lines, respectively. There are two main differences between this frame and a proper projective plane, however. First, $\mathcal{S}_{\mathbb{Q}}$ is one-sorted; this in itself can easily be modified by invoking the algebraic/geometric structure of the rational square: we may simply add all straight lines in the plane as line objects. However, we do not yet obtain a projective plane in this way; for instance, no two horizontal lines intersect. What we have to do is to add a new line, a horizon, or line at infinity where all lines intersect that were thus far parallel; obviously, by adding this new line, we also have to add a bunch of new points lying on this horizon.

Now that we have 'embedded' the rational square in this projective plane, we deal with the valuation. We will colour the vertical lines blue 0 and the horizontal lines blue ${ }_{1}$, respectively. This means that every old point will be incident with a light blue line of each shade. Then, our valuation will be such that the new line will be coloured both shades of blue. This already shows that the formula sky holds in the model. But also, the new line will be the only dark blue line; this means that old points are light. Since a horizontal and a vertical line can only intersect in an old point, and the new line is dark blue, no new points will be light. This shows that the formula but holds in the model.

Our construction is such that every old point is incident with a unique line of each blue shade; this shows that for every $\sigma$, every old point satisfies the formula $\langle 01\rangle\left(1 \mathrm{~b}_{i} \wedge\langle 10\rangle(\mathrm{light} \wedge\right.$ $\sigma)) \rightarrow[\cdot]\left(\mathrm{lb}_{i} \rightarrow\langle 10\rangle(\mathrm{light} \wedge \sigma)\right)$. Since the old points are the only light ones, we have that $\mathrm{bb}(\sigma)$ holds everywhere in the model. Finally, the whole construction is tailored towards the aim that any $L_{2}$-formula $\varphi$ is true in a rational pair iff $\varphi^{+}$is true at the corresponding point in the projective plane.

Now we go into the mathematical details. The projective plane that we have just been describing in words is in fact well-known as the rational projective plane. This plane can be more precisely described as follows. Consider the following relation $\sim$ on triples of rational numbers

$$
\left(q_{0}, q_{1}, q_{2}\right) \sim\left(r_{0}, r_{1}, r_{2}\right) \text { iff } \lambda q_{0}=r_{0}, \lambda q_{1}=r_{1} \text { and } \lambda q_{2}=r_{2} \text { for some } \lambda \neq 0 .
$$

This relation is obviously an equivalence relation; an equivalence class of this relation is called a ratio, at least, if it is not the singleton equivalence class of the triple $(0,0,0)$. Now the sets $P$ of points and $L$ of lines in $\mathcal{P}_{2}(\mathbb{Q})$ are both defined as the collection of ratios; to denote the difference between points and lines, we use $\left[q_{0}, q_{1}, q_{2}\right]$ as the point, and $\left\langle q_{0}, q_{1}, q_{2}\right\rangle$ as the line associated with the triple $\left(q_{0}, q_{1}, q_{2}\right)$. A point $\left[q_{0}, q_{1}, q_{2}\right]$ is incident with the line $\left\langle a_{0}, a_{1}, a_{2}\right\rangle$ if $a_{0} q_{0}+a_{1} q_{1}+a_{2} q_{2}=0$ - observe that this is well-defined. For a proof that $\mathcal{P}_{2}(\mathbb{Q})$ is in fact a projective plane, the reader can consult any text book on projective geometry (see for instance section 1.4 of HEYTING [3]. (This construction can in fact be carried out for every field.)

Before defining a valuation on $\mathcal{P}_{2}(\mathbb{Q})$, let us first see how the states of $\mathcal{M}$, that is, the rational pairs, can be represented as points in $\mathcal{P}_{2}(\mathbb{Q})$. Consider the following function mapping rational pairs to points of $\mathcal{P}_{2}(\mathbb{Q})$ :

$$
\left(q_{0}, q_{1}\right) \mapsto\left[q_{0}, q_{1}, 1\right]
$$


We leave it to the reader to verify that this is an injection; this observation does not play a role in the sequel. Let us now agree to call a point in $P$ old if it is of the form $\left[q_{0}, q_{1}, 1\right]$, that is, if it is in the range of the mapping above. Points that are not old are called new; it is easy to verify that any new point is of the form $\left[q_{0}, q_{1}, 0\right]$ for some rationals $q_{0}$ and $q_{1}$.

Consider the following valuation $V^{\prime}$ on $\mathcal{P}_{2}(\mathbb{Q})$ :

$$
\begin{aligned}
V^{\prime}(p) & :=\left\{\left[q_{0}, q_{1}, 1\right] \mid\left(q_{0}, q_{1}\right) \in V(p)\right\} \\
V^{\prime}\left(b_{i}\right) & :=\left\{\left\langle a_{0}, a_{1}, a_{2}\right\rangle \mid a_{1-i}=0\right\} .
\end{aligned}
$$

In order to show $\pi(\xi)$ to be satisfiable in the model $\mathcal{M}^{\prime}=\left(\mathcal{P}_{2}(\mathbb{Q}), V^{\prime}\right)$, we need the following claims.

Every point is incident with a blue $i$ line.

Let $s$ be some point of $\mathcal{P}_{2}(\mathbb{Q})$; we only treat the case that $i=1$. If $s=\left[q_{0}, q_{1}, 1\right]$ is old, take the line $\left\langle 0,1,-q_{1}\right\rangle$; if $s=\left[q_{0}, q_{1}, 0\right]$ is new, take the line $\langle 0,0,1\rangle$. In both cases we have found a blue ${ }_{1}$ line that is incident with $s$. This proves (1).

(2) Every old point is incident with a unique blue ${ }_{i}$ line, which is light blue . $_{\text {. }}$

Again, assume that $i=1$, and consider an arbitrary old point $\left[q_{0}, q_{1}, 1\right]$. The line $\left\langle 0,1,-q_{1}\right\rangle$, incident with $\left[q_{0}, q_{1}, 1\right]$ is not only blue ${ }_{1}$, but even light blue ${ }_{1}$; this proves existence. For unicity, suppose that the line $\left\langle 0, b_{1}, b_{2}\right\rangle$ is also incident with $\left[q_{0}, q_{1}, 1\right]$. By definition, we find that $b_{1} q_{1}+b_{2}=0$. But then $b_{2}=b_{1}\left(-q_{1}\right)$, so $\left(0, b_{1}, b_{2}\right) \sim\left(0,1,-q_{1}\right)$. This means that $\left\langle 0, b_{1}, b_{2}\right\rangle$ is in fact identical to $\left\langle 0,1,-q_{1}\right\rangle$, and thus proves $(2)$.

Any point $s$ of $\mathcal{P}_{2}(\mathbb{Q})$ is old iff $\mathcal{M}, s \Vdash$ light.

If $s$ is new, say $s=\left[q_{0}, q_{1}, 0\right]$, then $s$ is incident with the line $\langle 0,0,1\rangle$ which is dark blue. Hence, $\mathcal{M}^{\prime}, s \mid \forall$ light.

For the other direction, suppose that $\mathcal{M}^{\prime},\left[q_{0}, q_{1}, q_{2}\right] \forall$ light. Let $\left\langle a_{0}, a_{1}, a_{2}\right\rangle$ be a dark blue line through $\left[q_{0}, q_{1}, q_{2}\right]$. It follows by the definition of the valuation that $a_{0}=a_{1}=0$; since $\left(a_{0}, a_{1}, a_{2}\right)$ is a ratio, this means that $a_{2} \neq 0$. But by the definition of the incidence relation, we have that $a_{2} q_{2}=0$. This can only be the case if $q_{2}=0$. But then $\left[q_{0}, q_{1}, q_{2}\right]$ is new. This proves (3).

No new point is incident with a light blue $i$ line of each shade.

Observe that a light blue $e_{0}$ line must be of the form $\left\langle 1,0, a_{2}\right\rangle$, and a light blue ${ }_{1}$ line of the form $\left\langle 0,1, b_{2}\right\rangle$. Now, in order to derive a contradiction, suppose that the new point $\left[q_{0}, q_{1}, 0\right]$ is incident with both $\left\langle 1,0, a_{2}\right\rangle$ and $\left\langle 0,1, b_{2}\right\rangle$. By definition of the incidence relation, we get $q_{0}=0$ and $q_{1}=0$. But then $\left(q_{0}, q_{1}, 0\right)$ is not a ratio. This proves $(4)$.

For all $\left(q_{0}, q_{1}\right),\left(r_{0}, r_{1}\right)$ in $\mathbb{Q}^{2}$ :

$\left(q_{0}, q_{1}\right) \equiv_{i}\left(r_{0}, r_{1}\right)$ iff some light blue $i$ line connects $\left[q_{0}, q_{1}, 1\right]$ and $\left[r_{0}, r_{1}, 1\right]$. 
We only treat the case where $i=1$. First assume that $\left(q_{0}, q_{1}\right) \equiv_{1}\left(r_{0}, r_{1}\right)$; that is, $\left(r_{0}, r_{1}\right)=$ $\left(q_{0}^{\prime}, q_{1}\right)$ for some $q_{0}^{\prime} \in \mathbb{Q}$. It is straightforward to verify that the line $\left\langle 0,-1, q_{1}\right\rangle$ connects $\left[q_{0}, q_{1}, 1\right]$ and $\left[q_{0}^{\prime}, q_{1}, 1\right]$.

For the other direction, suppose that the light blue ${ }_{1}$ line $\left\langle a_{0}, a_{1}, a_{2}\right\rangle$ connects the points $\left[q_{0}, q_{1}, 1\right]$ and $\left[r_{0}, r_{1}, 1\right]$. Since $\left\langle a_{0}, a_{1}, a_{2}\right\rangle$ is light blue ${ }_{1}$, we have $a_{0}=0$ and $a_{1} \neq 0$. By definition of incidence, this gives

$$
\left\{\begin{array}{l}
a_{1} q_{1}+a_{2}=0 \\
a_{1} r_{1}+a_{2}=0
\end{array}\right.
$$

But then it is immediate that $q_{1}=-a_{2} / a_{1}=r_{1}$. This shows that $\left(q_{0}, q_{1}\right) \equiv_{1}\left(r_{0}, r_{1}\right)$, and proves (5).

(6) For any $\left(q_{0}, q_{1}\right) \in \mathbb{Q}^{2}$, and any $L_{2}$-formula $\varphi$ : $\mathcal{M},\left(q_{0}, q_{1}\right) \Vdash \varphi$ iff $\mathcal{M}^{\prime},\left[q_{0}, q_{1}, 1\right] \Vdash \varphi^{+}$.

The proof of (6) is by a straightforward induction on the complexity of $\varphi$. We only treat one modal case of the inductive step: assume that $\varphi$ is of the form $\diamond_{0} \psi$.

First assume that $\mathcal{M},\left(q_{0}, q_{1}\right) \Vdash \varphi$. Hence, there is some $q_{1}^{\prime} \in \mathbb{Q}$ such that $\mathcal{M},\left(q_{0}, q_{1}^{\prime}\right) \Vdash \psi$. By the inductive hypothesis, this implies that $\mathcal{M}^{\prime},\left[q_{0}, q_{1}^{\prime}, 1\right] \Vdash \psi^{+}$. By (5), there is some light blue $e_{0}$ line connecting $\left[q_{0}, q_{1}, 1\right]$ and $\left[q_{0}, q_{1}^{\prime}, 1\right]$. But then using $(3)$ it is easy to verify that $\mathcal{M}^{\prime},\left[q_{0}, q_{1}, 1\right] \Vdash\langle\cdot\rangle_{0} \psi^{+}$; or equivalently, that $\mathcal{M}^{\prime},\left[q_{0}, q_{1}, 1\right] \Vdash \varphi^{+}$.

For the other direction, assume that $\mathcal{M}^{\prime},\left[q_{0}, q_{1}, 1\right] \Vdash\langle\cdot\rangle_{0} \psi^{+}$. This implies the existence of a line $k$ and a point $s$ such that $k$ is incident with both $\left[q_{0}, q_{1}, 1\right]$ and $s, \mathcal{M}^{\prime}, k \Vdash 1 \mathrm{~b}_{0}$, and $\mathcal{M}^{\prime}, s \Vdash$ light $\wedge \psi^{+}$. But by (3), $s$ is old if it is light, say $s=\left\langle r_{0}, r_{1}, 1\right\rangle$. It follows from (5) that $r_{0}=q_{0}$, and from the inductive hypothesis, that $\mathcal{M},\left(r_{0}, r_{1}\right) \Vdash \psi$. Bringing these observations together we find that $\mathcal{M},\left(q_{0}, q_{1}\right) \Vdash \diamond_{0} \psi$. This proves (6).

We have now sufficient information to prove that $\pi(\xi)$ is satisfiable in $\mathcal{M}^{\prime}$. Let $\left(q_{0}, q_{1}\right)$ be the rational pair in $\mathcal{M}$ where $\xi$ holds. It follows from (6) that $\mathcal{M}^{\prime},\left[q_{0}, q_{1}, 1\right] \Vdash \xi^{+}$and from (3) that $\mathcal{M}^{\prime},\left[q_{0}, q_{1}, 1\right] \Vdash$ light. From (1) we immediately infer that $\mathcal{M}^{\prime},\left[q_{0}, q_{1}, 1\right] \Vdash$ sky.

Now consider an arbitrary point $s$ of $\mathcal{M}^{\prime}$. If $s$ is old, then $\mathcal{M}^{\prime}, s \Vdash$ light by (3); and if $s$ is new, $\left.\mathcal{M}^{\prime}, s \Vdash \neg(\langle 01\rangle] \mathrm{b}_{0} \wedge\langle 01\rangle \mathrm{l} \mathrm{b}_{1}\right)$ by (4). In both cases we find that $\mathcal{M}^{\prime}, s \Vdash(\langle 01\rangle] \mathrm{b}_{0} \wedge$ $\left.\langle 01\rangle \mathrm{lb}_{1}\right) \rightarrow$ light; thus $\mathcal{M}^{\prime},\left[q_{0}, q_{1}, 1\right] \Vdash$ but.

Finally, it is immediate by $(2)$ that $\mathcal{M}^{\prime}, s \Vdash \bigwedge_{i}\left(\left(\right.\right.$ light $\left.\wedge\langle\cdot\rangle_{i} \sigma\right) \rightarrow[01]\left(\mathrm{lb}_{i} \rightarrow\langle 10\rangle(\right.$ light $\wedge$ $\sigma))$ ) for any point formula $\sigma$ and any light point $s$. This proves that $\mathcal{M}^{\prime},\left[q_{0}, q_{1}, 1\right] \Vdash$ $\bigwedge_{\varphi \in C l(\xi)}[\cdot] \mathrm{bb}\left(\varphi^{+}\right)$.

Thus we have shown that each of the conjuncts of $\pi(\xi)$ holds at $\left[q_{0}, q_{1}, 1\right]$, whence $\pi(\xi)$ is indeed satisfiable in $\mathcal{P}_{2}(\mathbb{Q})$.

Claim 2 An $L_{2}$-formula $\xi$ is satisfiable in $\mathrm{K}$ if $\pi(\xi)$ is satisfiable in PP.

Proof of Claim Assume that $\pi(\xi)$ is satisfiable in the model $\mathcal{M}=(P, L, I, V)$ where $\mathcal{G}=(P, L, I)$ is some projective plane. We will define a model $\mathcal{M}^{\prime}$ over some $\mathrm{K}$-frame $\mathcal{F}=$ $\left(W, R_{0}, R_{1}\right)$ in which $\xi$ is satisfiable. 
We use the following terminology. For a point $t$ in $\mathcal{M}$, let $\Omega_{t}$ denote the set of $L_{2}$-formulas $\varphi \in C l(\xi)$ such that $\mathcal{M}, t \Vdash \varphi^{+}$. Now define $W, R_{i}$ and $V^{\prime}$ as follows:

$$
\begin{aligned}
W & :=\left\{\Omega_{t} \mid t \in P \text { for some light } t\right\} \\
R_{i} & :=\left\{(\Gamma, \Delta) \in W^{2} \mid \text { for all } \varphi \in C l(\xi): \diamond_{i} \varphi \in \Gamma \text { iff } \diamond_{i} \varphi \in \Delta\right\} . \\
V(p) & :=\{\Gamma \in W \mid p \in \Gamma\}
\end{aligned}
$$

The crucial observation underlying our definitions is the following.

$$
\text { If two light points } t \text { and } u \text { are connected by a blue } i \text { line, then } \Omega_{t} R_{i} \Omega_{u} \text {. }
$$

Assume that $l$ is a blue $i$ line connecting the light points $t$ and $u$. Observe that $l$ must be light blue $_{i}$, otherwise $t$ and $u$ would not be light.

Now assume that $\diamond_{i} \varphi$ is a subformula of $\xi$ that belongs to $\Omega_{t}$. By definition of $\Omega_{t}$, this means that $\mathcal{M}, t \Vdash\langle\cdot\rangle_{i} \varphi^{+}$. But since $\pi(\xi)$ is satisfiable in $\mathcal{M}$, we have that $\mathcal{M}, t \Vdash \operatorname{bb}\left(\varphi^{+}\right)$. This implies that

$$
\mathcal{M}, t \Vdash[01]\left(\mathrm{lb}_{i} \rightarrow\langle 10\rangle\left(\text { light } \wedge \varphi^{+}\right)\right) .
$$

Now $l$ is a lightblue $i$ line through $t$; hence, we have that $\mathcal{M}, l \Vdash\langle 10\rangle\left(\right.$ light $\left.\wedge \varphi^{+}\right)$. But the fact that $u$ lies on $l$ as well implies that $\mathcal{M}, u \Vdash\langle\cdot\rangle_{i} \varphi^{+}$. This gives that $\diamond_{i} \varphi$ belongs to $\Omega_{u}$.

With a similar argument we can show that any formula $\diamond_{i} \varphi \in \Omega_{u}$ must belong to $\Omega_{t}$ as well. But that shows that $\Omega_{t} R_{i} \Omega_{u}$, and proves (7).

Our first aim is now to show the following.

$$
\mathcal{F} \text { belongs to } \mathrm{K} \text {. }
$$

It follows immediately from the definitions that each $R_{i}$ is an equivalence relation, so we only have to show that $R_{0}$ and $R_{1}$ commute. Let $\Gamma, \Delta$ and $\Theta$ be elements of $W$ such that $\Gamma R_{0} \Delta R_{1} \Theta$. We have to find a $\Lambda$ such that $\Gamma R_{1} \Lambda R_{1} \Theta$. By definition of $W$, there are light points $t$ and $u$ such that $\Gamma=\Omega_{t}, \Delta=\Omega_{u}$. It follows from the satisfiability of $\pi(\xi)$ in $\mathcal{M}$ (in particular, from the fact that sky holds at each point in $\mathcal{M}$ ) that there is a blue 0 line $k$ through $t$ and a blue ${ }_{1}$ line $l$ through $u$. Since $t$ and $u$ are light, $k$ and $l$ cannot be dark blue; it follows that $k$ is light blue 0 and $l$ is light blue .

Now $\mathcal{G}$ is a projective plane, so $k$ and $l$ intersect in some point $v$. It follows from $\mathcal{M}, v \Vdash$ but that $v$ is light. But then by applying (7) we see that $\Omega_{t} R_{0} \Omega_{v}$ and $\Omega_{u} R_{1} \Omega_{v}$. In other words, $\Omega_{v}$ is the $\Lambda$ that we were looking for. This proves (8).

Finally, we prove a truth lemma for $\mathcal{M}^{\prime}$.

For all $\varphi \in C l(\xi)$ and all $\Gamma \in W: \mathcal{M}^{\prime}, \Gamma \Vdash \varphi$ iff $\varphi \in \Gamma$.

Obviously, we prove (9) by induction on the complexity of $\varphi$. We concentrate on the modal case of the inductive step: assume that $\varphi$ is of the form $\diamond_{i} \psi$.

First assume that $\mathcal{M}^{\prime}, \Gamma \Vdash \diamond_{i} \psi$. By the truth definition, there is some $\Delta$ in $W$ with $\Gamma R_{i} \Delta$ and $\mathcal{M}^{\prime}, \Delta \Vdash \psi$. By definition of $W$ there is some light point $t$ such that $\Delta=\Omega_{t}$, and by definition of $\Omega_{t}$, we have that $\mathcal{M}, t \Vdash \psi^{+}$. It follows from the satisfiability of $\pi(\xi)$ in $\mathcal{M}$ 
(again, use the sky-conjunct) that any point is incident with some blue ${ }_{i}$ line; and since $t$ is light, we know that $t$ is incident with some light blue ${ }_{i}$ line $k$. But then $t$ sees itself along a light blue ${ }_{i}$ line, so from $\mathcal{M}, t \Vdash \psi^{+}$it follows that $\mathcal{M}, t \Vdash\langle\cdot\rangle_{i} \psi^{+}$; hence, by definition of $\Omega_{t}$ we obtain that $\diamond_{i} \psi \in \Omega_{t}=\Delta$. But then from $\Gamma R_{i} \Delta$ we may conclude that $\varphi=\diamond_{i} \psi \in \Gamma$.

For the other direction, assume that $\diamond_{i} \psi \in \Gamma$. It follows by the definition of $W$ that $\Gamma=\Omega_{s}$ for some light point $s$; and from the definition of $\Omega_{s}$, that $\mathcal{M}, s \Vdash\langle\cdot\rangle_{i} \psi^{+}$. We can then use the truth definition to find a light blue ${ }_{i}$ line $l$ and a light point $t$ on $l$ such that $\mathcal{M}, t \Vdash \psi^{+}$. By definition of $\Omega_{t}$ we see that $\psi \in \Omega_{t}$, so by the inductive hypothesis we obtain $\mathcal{M}^{\prime}, \Omega_{t} \Vdash \psi$. But from (7) it follows that $\Gamma R_{i} \Omega_{t}$. Taking these observation together, we find that $\mathcal{M}^{\prime}, \Gamma \Vdash \diamond_{i} \psi$. This proves $(9)$.

Finally, it follows from (9) and the satisfiability of $\pi(\xi)$ in $\mathcal{M}$ that $\xi$ is satisfiable in $\mathcal{M}^{\prime}$. But then the claim is immediate by (8).

\section{References}

[1] Ph. Balbiani. The modal multilogic of geometry. Journal of Applied Non-Classical Logics, 8:259-281, 1998.

[2] Ph. Balbiani, L. Fariñas del Cerro, T. Tinchev, and D. Vakarelov. Modal logics for incidence geometries. Journal of Logic and Computation, 7:59-78, 1997.

[3] A. Heyting. Axiomatic Projective Geometry. P. Noordhof N.V., Groningen, 1963.

[4] O. Lemon. Semantical foundations of spatial logics. In Principles of Knowledge Representation and Reasoning: Proceedings of the Fifth International Conference (KR '96), pages 212-219. Morgan Kaufmann Publisherss, 1996.

[5] O. Lemon and I. Pratt. On the incompleteness of modal logics of space: advancing complete modal logics of space. In Advances in Modal Logic '96. CSLI Publications, to appear.

[6] M. Marx. Complexity of modal logics of relations. Technical Report ML-97-02, Institute for Logic, Language and Computation, University of Amsterdam, 1997.

[7] M. Marx and Y. Venema. Multi-Dimensional Modal Logic. Kluwer Academic Publishers, Dordrecht, 1997.

[8] V. Stebletsova. Modal logic of projective geometries of finite dimension. Technical Report 184, Department of Philosophy, Utrecht University, 1998.

[9] Y. Venema. Cylindric modal logic. Journal of Symbolic Logic, 60:591-623, 1995. 